\title{
Research on Intelligent Computer Security Monitoring Information Network Technology
}

\author{
Che Liu, Yunpeng Wu \\ School of Information Engineering, Zhengzhou University, Zhengzhou, Henan, 45000, China
}

Keywords: Intelligent computer, Security monitoring, Information network technology

\begin{abstract}
With the rapid development of the Internet, computers have gradually become an indispensable auxiliary tool for all walks of life. However, due to the development and sharing of networks, it is difficult to implement all-round security monitoring. Therefore, it is necessary to strengthen the research on intelligent computer security monitoring information network technology and find out more effective, scientific and advanced intelligent computer security monitoring methods. It is necessary to take necessary measures to prevent and eliminate these security risks. Therefore, in practical work, it is necessary to conduct privacy processing of information through certain protection methods. The security monitoring information network technology of intelligent computer can directly monitor the information, ensure the security of information, prevent the invasion of foreign viruses, and make the computer network environment of our country more secure. This paper makes the following discussion on the preventive measures.
\end{abstract}

\section{Introduction}

At present, with the continuous development of the level of computers and science and technology, computers have become an indispensable auxiliary tool in all walks of life [1]. At the current stage, the current computer security monitoring technology has not yet set detailed security monitoring indicators. Therefore, the weight of non-security factors cannot be accurately calculated, which can easily lead to various security accidents [2]. For this reason, research on intelligent computer security monitoring information network technology should be strengthened to find more effective, scientific, and advanced methods for intelligent computer security monitoring [3]. At the same time, computer monitoring technology came into being, but it can not completely realize the monitoring and prevention of computer security, while the development of intelligent computer provides more effective monitoring and control, and further realizes the thorough monitoring and control of computer security issues [4].

The current computer security monitoring technology has not yet set specific security monitoring standards, therefore, the weight ratio of dangerous factors cannot be accurately calculated, which easily leads to many security accidents, such as automatic disclosure of secrets, relatively large errors in monitoring data, and failure to achieve intelligent monitoring effect [6]. Therefore, in practical work, information needs to be treated with privacy through certain protection methods. The security monitoring information network technology of intelligent computers can directly monitor information to ensure the safety of information, prevent the invasion of foreign viruses and make the computer network environment in our country safer [7].

\section{The Development Status of Intelligent Computer Security Monitoring Information Network Technology}

\subsection{Disadvantages and Advantages of Current Common Computer Security Monitoring Information Network Technology}

Computer security monitoring technology is a kind of technology to monitor, collect and record data and control computers. Its main function is to improve the security of computer users' information and to provide the advantages of information privacy. However, there are some 
inevitable problems in monitoring the security of computers, which greatly affect the design Computer security. Because of the great sharing and development of computer network, it is very easy to be restricted by various security risk conditions, which poses a certain threat to its security. In order to achieve the goal of intelligent security monitoring of computers, the existing security risks of computer networks should be distinguished. Intelligent computer is a technology that personifies computers. It mainly adds the application of artificial intelligence to the common computer security monitoring information network technology, realizes the intellectualization of computer monitoring technology, greatly improves the performance of the original monitoring system, expands the scope of monitoring computer security issues, and can also prolong the service life of computers to a certain extent. This also ensures more effective computer security monitoring.

\subsection{Current Situation of Intelligent Computer in Security Monitoring Information Network Technology}

Intelligent computer security monitoring is of great significance to the further development of computers. For this reason, it has become the focus of researchers on intelligent computer security monitoring information network technology. After a long period of continuous research, scholars in the field have made many achievements in computer security intelligent monitoring methods. Some researchers have proposed an intelligent computer security monitoring method based on GPRS. With the help of the remote network monitoring system, the real-time security monitoring of the computer can be realized. At the same time, the monitoring code is applied to mark the non security areas in the computer network. By using the communication link of the computer terminal, the non security information in the non security areas is submitted to the relevant management personnel, and then the management personnel carry out the security remedy for the computer. By adopting such a remote control method, the functional security monitoring of the computer can be realized, and the unsafe information existing in the computer network can be obtained in real time. However, this method can only implement remote monitoring, but cannot play the role of risk assessment. Therefore, it is easy to cause large or small differences in the security monitoring results of intelligent computers [8]. Related researchers have concluded an effective method for intelligent computer security monitoring on the premise of creating a "Web Service". This method is mainly to set up effective safety inspection devices at the entrance of computer information network and the exit of computer information network respectively with the aid of DFNMS system, so as to filter the incoming data information and outgoing data information in the computer network. Through effective analysis of this part of data, unsafe or risky information can be found out. After the method is implemented, the computer is intelligently monitored for safety. This method can prevent the submission of confidential content data in time and reduce the probability of active disclosure to a certain extent. However, this method needs to set up safety inspection devices at the entrance of the computer information network and the exit of the computer information network respectively, which is relatively high in cost. At the same time, regular maintenance inspection is required, which increases the maintenance inspection cost and wastes time.

\section{Research on Intelligent Computer Security Monitoring Information Network Technology}

\subsection{Construction of Computer Intelligent Security Monitoring Platform}

In view of these security risk factors that the computer network faces, a computer intelligent security monitoring platform is constructed, and the security monitoring of each part of the computer is carefully divided. The platform is mainly divided into four modules, namely, the network risk assessment module, the network security alarm module, the network security emergency module and the network remote security intelligent monitoring module. The network risk assessment is mainly to assess the risks in computers and the functions of full emergency and security alarm with risks. The second part, the network security alarm is mainly to warn the danger and risk factors in the computer, so as to ensure the intelligent security monitoring system of the computer to maintain its visible characteristics. The third part, the network security emergency is 
mainly to deal with the unexpected situation in the computer intelligent security monitoring timely and effectively, so as to prevent the computer from being unable to use or data information damage and loss. The fourth part, in a specific environment, if the computer can not implement intelligent security monitoring in this area, then remote intelligent security monitoring can be used to carry out intelligent security monitoring on the computer. Constructing a computer intelligent security monitoring network and implementing three-dimensional and comprehensive intelligent security monitoring for computers is conducive to ensuring the security of computer networks, which is of great significance for promoting the development of computers.

In order to further verify the overall performance of the computer security intelligent monitoring method based on Linux, Table 1 compares the average risk security assessment rate (\%) of computer security intelligent monitoring. As shown in the Figure1, the evaluation rate of the three research methods changes with time.

Table 1 Evaluation Rate Of Intelligent Monitoring Risk Security

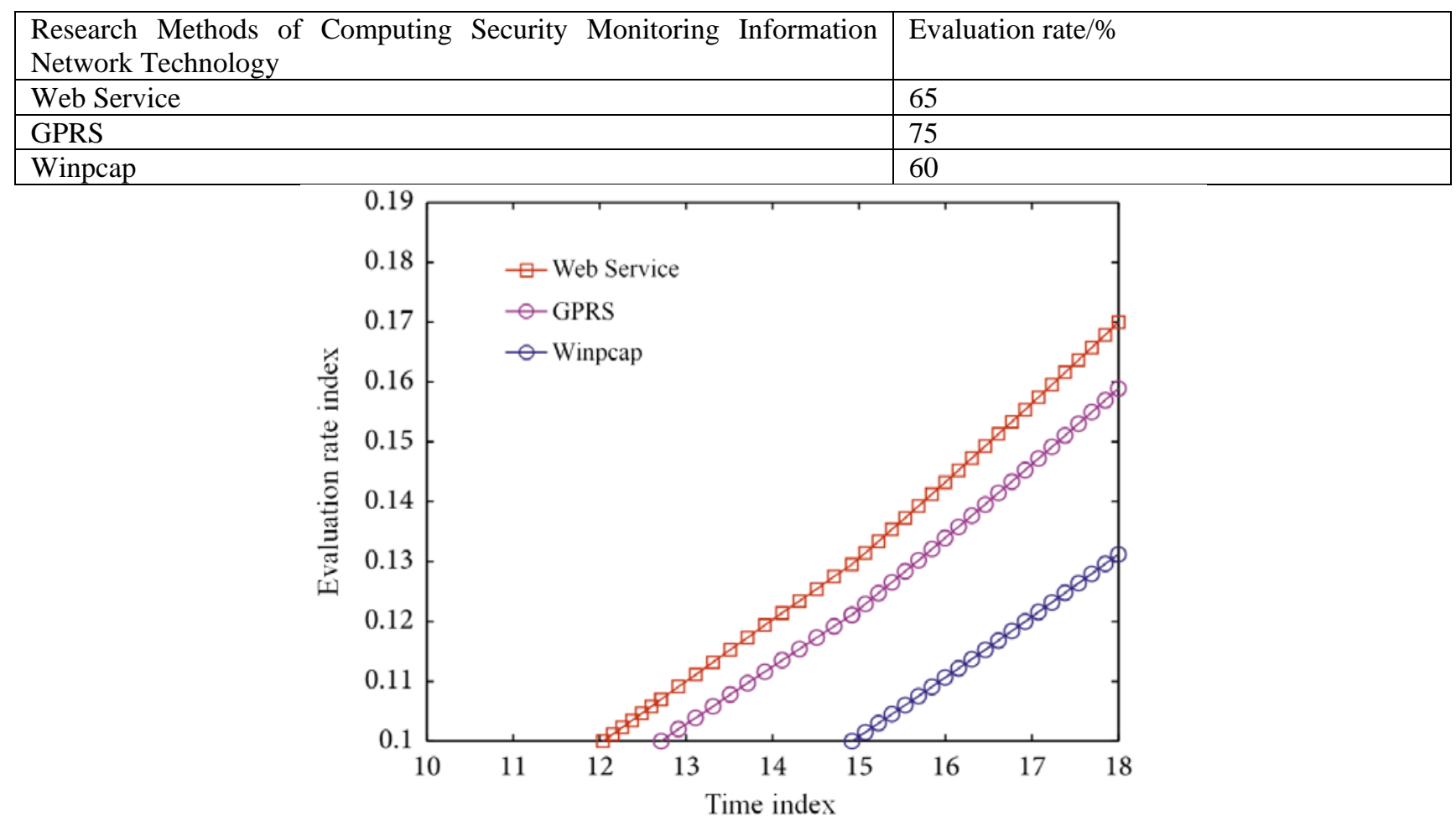

Fig.1 Changes in the Risk Assessment Rate of Intelligent Monitoring Risks

\subsection{Research on Key Points Related to Intelligent Computer Security Monitoring Information Network Technology}

In design, the characteristics of intelligent computer monitoring technology to achieve intelligent computer security monitoring, first of all, to understand the computer network security is vulnerable to which network security factors interference, and to classify these factors, more convenient for intelligent computer security monitoring. The intelligent monitoring network is established to monitor the computer security problems in all aspects, and it also realizes the three-dimensional monitoring, effectively strengthens the computer security technology, and can solve these security risks in time. In practice, the intelligent computer monitoring technology shows the superiority, and the intelligent computer security monitoring technology adopts the Linux intelligent computer security The monitoring method, however, needs to be improved according to the large-scale real data statistics results. So far, a large number of simulation experiments have been carried out on the intelligent computer monitoring technology. With the assistance of MATLAB 2016a, a platform with a higher degree of simulation can be established. The experimental data was obtained from 100 computers in the libpcap system. At the same time, it was mixed with a variety of risk factors that are not of a network nature, such as information related to harassment, information related to fraud, 
and some related information. Fraudulent websites, malicious software, information leakage, account theft, viruses, or Trojans and other factors. Check the comprehensive performance of this intelligent computer security monitoring method over a period of time.

When the computer security intelligent monitoring method based on LINUX is used for intelligent monitoring, the monitoring rate (\%) of computer security problems found in the intelligent monitoring is shown in Table 2. The data in this table determine the comprehensive effectiveness of the computer security intelligent monitoring method based on linux.

Table 2 Monitoring Rate of Security Problems Found by Intelligent Monitoring

\begin{tabular}{|l|l|}
\hline Problem type / species & Incidence/\% \\
\hline Harassment Information & 77 \\
\hline Fraud information & 75 \\
\hline Malicious software & 76 \\
\hline Scam sites & 85 \\
\hline Poisoning or Trojan & 81 \\
\hline Information leakage & 80 \\
\hline Account stolen & 77 \\
\hline Other & 70 \\
\hline
\end{tabular}

It can be seen from table 1 that when the computer security intelligent monitoring method based on Linux performs the security intelligent monitoring, the monitoring rate of harassment information is $77 \%$, the monitoring rate of fraud information is $75 \%$, the monitoring rate of malware is $76 \%$, the monitoring rate of fraud website is $85 \%$, the monitoring rate of poisoning or Trojan horse is $81 \%$, the monitoring rate of information leakage is $80 \%$, and the monitoring rate of account theft is $80 \%, 77 \%$ and $70 \%$ for other monitoring. The experimental results show that the proposed method can effectively monitor the computer security and intelligence.

\subsection{Development Prospect of Intelligent Computer Security Monitoring Information Network Technology}

Although intelligent computer monitoring technology achieves a more comprehensive and effective superiority than ordinary computer monitoring technology to a certain extent, it cannot meet the comprehensive requirements. The answer to monitoring the computer as a whole is a question mark This will also be an inevitable requirement for the development of the Internet to solve the problem of computer security monitoring. Because intelligent monitoring of computer security is of great significance to the development of computers, it has become the focus of the researchers of intelligent monitoring technology of computer network security, and has received great attention. This also requires the relevant departments and national readers to provide strong technical support to ensure that the intelligent computing monitoring technology is more comprehensive, more integrated and more effective to achieve the monitoring of computer security.

\section{Conclusions}

In our country, whether it is an enterprise department or a government department, it is necessary to complete the daily work through the information transmission of computers. The computer security monitoring system is the security guarantee for completing the work, and it is also the direct way for enterprises or government departments to monitor the information exchange of staff. In order to realize the monitoring of information texts and files at the same time, our country keeps updating new intelligent technologies to ensure the direct operation of information network technology. The implementation of computer security monitoring is to ensure the safety of computer information and secret transmission, as well as the user's use safety and property safety, which is a technical means to benefit the country and the people. This also needs to be taken seriously, invest in vigorously developing this technology, improve this technology, realize the intelligent computer security monitoring as soon as possible, protect the interests of the majority of computer users, promote the more secure and rapid development of the Internet cause, and bring more More convenience. 


\section{References}

[1] Zhang Ruirui. (2018). Research and application of computer network security prevention technology. Science and Technology Innovation and Application, no. 35, pp. 179-180.

[2] Liu Chibiao, Wu Jun, Zhang Minghua, Lu Yongdong, Chen Bin, \& Huang Baojing, et al. (2013). Development of Intelligent Network Examination and Monitoring System. Network Security Technology and Application, no. 07, pp. 92-94.

[3] Zhang Jie. (2013). Computer digital network simulation application based on building intelligent technology. Information Communication, no. 10, pp. 87-88.

[4] Zhou Li. (2017). Design and implementation of computer intelligent network monitoring system. Digital World, no. 10, pp. 246-247.

[5] Xie Yangui, \& Zhong Shaodan. (2010). Design and Implementation of Computer Intelligent Network Monitoring System. Modern Computer (Professional Edition), no. 6, pp. 196-197.

[6] Yang Kunchao. (2016). Computer Network Information Management and Its Security Protection Strategy. Global Market Information Review, no. 42, pp. 125-125.

[7] Hou Minghao. (2016). Research on Computer Network Security Monitoring and Key Technologies. Electronic Technology and Software Engineering, no. 10, pp. 231-231.

[8] Tu Ruliang. (2017). Design and implementation of computer intelligent network monitoring system. Electronic Technology and Software Engineering, no.10, pp. 10-10.

[9] Chen Weiwei. (2018). Design and Analysis of Computer Network Monitoring System Based on Intelligent Technology. Automation Technology and Application, no. 10, pp. 159-162. 